Die Flächenverhältnisse der identifizierten Banden in den hochsiedenden Anteilen sind in Tab. 5 b zusammengestellt.

Bei der Umsetzung der Diphenylalkane befand sich kurz vor dem Entladungsrohr ein mit Heißluft beheizter Kolben mit der Substanz. Die Zusammensetzung der entstandenen Destillate ist in Tab. 6 a wiedergegeben.

Höhersiedende Produkte schieden sich zusammen mit nichtumgesetztem Material am Ende des Entladungs- rohres ab. In Tab. $6 \mathrm{~b}$ sind die in den höhersiedenden Anteilen nachgewiesenen Produkte zusammengestellt.

Der Deutschen Forschungsgemeinschaft und dem Fonds der Chemischen Industrie danke ich für die finanzielle Unterstützung der Arbeit, Herrn Dr. W. KöNıg für die Aufnahme der Massenspektren, Herrn Dr. K. Scheffler für die Überlassung einer Mikrowellenapparatur.

Zum Wirkungsmechanismus von 1-Nitroso-3-nitro-1-methyl-guanidin bei der Mutationsauslösung

\title{
II. Produkte der Reaktion von 1-Nitroso-3-nitro-1-methyl-guanidin mit Nucleobasen, Nucleosiden, Nucleosidphosphaten und Homopolyribo- nucleinsäuren
}

\author{
F. Lingens, J. Rau und R. Süssmuth
}

Institut für Mikrobiologie und Molekularbiologie der Universität Hohenheim (Stuttgart-Hohenheim)

(Z. Naturforschg. 23 b, 1565-1570 [1968] ; eingegangen am 26. Juli 1968)

\begin{abstract}
1-Nitroso-3-nitro-1-methyl-guanidine (NNMG) (at pH 5,5 resp. 6,0) methylates and deaminates the bases of nucleic acids. We treated nucleic bases, nucleosides, nucleoside phosphates and homopolyribonucleic acids with NNMG and we found the following methyl derivates of adenine: 1-methyladenine, 3-methyladenine and $N^{6}$-methyladenine: of guanine: 7-methylguanine and of cytosine: 1-methylcytosine and 3-methylcytosine. Moreover bases of the nucleic acids were deaminated: adenine to hypoxanthine and guanine to xanthine.
\end{abstract}

In der ersten Mitteilung ${ }^{1}$ haben wir über die Wirkung von 1-Nitroso-3-nitro-1-methyl-guanidin (NNMG) auf die Matrizenaktivität der Polynucleotide bei der zellfreien Proteinsynthese berichtet.

Für die Mutationsauslösung durch NNMG ${ }^{2-8}$ kommen vor allem 3 Reaktionen mit den Nucleobasen in Frage: die Methylierung ${ }^{1,9,10}$, die Desaminierung ${ }^{1,11}$ und die Nitroguanylierung. Wir haben Methylierung und Desaminierung hauptsächlich unter ähnlichen Bedingungen (bei $37^{\circ}$ oder Zimmertemperatur und $\mathrm{pH} 5,5$ bzw. 6,0) untersucht, wie sie bei der Mutationsauslösung vorliegen.

1 P. Chandra, A. Wacker, R. Süssmuth u. F. Lingens, Z. Naturforschg. 22 b, 512 [1967].

2 J. D. Mandell u. J. Greenberg, Biochem. biophysic. Res. Commun. 3, 575 [1960].

3 J. D. Mandell, P. L. Woody u. J. Greenberg, J. Bacteriol. 81, 149 [1961].

4 A. J. Müller u. T. Gichner, Nature [London] 201, 1149 [1964].

5 R. Schwaier, Z. Vererbungslehre 97, 55 [1965].

\section{Material und Methoden}

Citronensäure-Phosphat-Puffer nach $\mathrm{M} \mathrm{c} \mathrm{Ilva} \mathrm{in} \mathrm{e:}$ Gemisch von $0,2-m$. $\mathrm{Na}_{2} \mathrm{HPO}_{4}$ und $0,1-m$. Citronensäure.

Zur Dünnschichtchromatographie verwendete Laufmittel:

S1 : n-Butanol/Eisessig/Wasser (4/1/1), S2: Methanol/konz. Salzsäure/Wasser $(7 / 2 / 1), \mathrm{S} 3: 3 \% \mathrm{NH}_{4} \mathrm{Cl} /$ $\mathrm{H}_{2} \mathrm{O}, \mathrm{S} 4$ : Isopropanol/Wasser/Ammoniak $(8 / 1 / 1)$, S5: n-Butanol/Wasser (1/11), S6: Isopropanol/konz. Salzsäure/Wasser (65/16/19), S7: Isopropanol/Wasser (6/4), S8: n-Buthanol/Äthanol/Wasser $(5 / 2 / 3)$, S9: n-Butanol (wassergesättigt)/Ammoniak (100/1), S10: Äthanol/Wasser/Ammoniak (80/8/2).

${ }^{6}$ F. Lingens u. O. Oltmanns, Z. Naturforschg. 21 b, 660 [1966].

7 D. R. McCalla, Science [Washington] 148, 497 [1965].

8 H. B. Singer u. H. Fraenkel-Conrat, Proc. nat. Akad. Sci. USA 58, 234 [1967].

9 J. Rau u. F. Lingens, Naturwissenschaften 54, 517 [1967].

10 F. Lingens, R. Süssmuth, A. Wacker u. P. Chandra, Naturwissenschaften 54, 492 [1967].

11 F. K. Zimmermann, R. Schwaier u. U. v. Laer, Z. Vererbungslehre 97, 68 [1965]. 
Umsetzungen von NNMG mit Nucleobasen und ihren Derivaten

Adenin: $20,7 \mathrm{mg}(0,15 \mathrm{mMol})$ Adenin wurden in $4,4 \mathrm{ml}$ Puffergemisch vom $\mathrm{pH} 5,5$ bei $80^{\circ} \mathrm{C}$ gelöst und dazu $218 \mathrm{mg} \quad(1,5 \mathrm{mMol})$ NNMG gegeben. Nach 34 Stdn. ist das Reaktionsgemisch an Dowex 1X8 (100 bis 200 mesh, $\left.\mathrm{Cl}^{\ominus} / \mathrm{H}_{2} \mathrm{O}, 1,5 \cdot 20 \mathrm{~cm}\right)$ getrennt worden: je 150 Tropfen $(10 \mathrm{ml}) \mathrm{NH}_{3}$-Lösung von $\mathrm{pH} 10$, wodurch Adenin zurückgehalten wird. Fraktionen $1-20$ wurden an Dowex $50 \mathrm{WX} 12\left(200-400 \mathrm{mesh}, \mathrm{H}^{\oplus} / \mathrm{H}_{2} \mathrm{O}\right.$, $27 \cdot 7 \mathrm{~cm})$ wiederholt getrennt. Je $150 \mathrm{Tr} . \mathrm{H}_{2} \mathrm{O}$ Fr. 101 bis 120 (die Guanidine laufen durch. Nucleobasen werden zurückgehalten), 2-n. HCl Fr. 121 ff.

Dünnschichtchromatographie an MN 400

$\begin{array}{lccc}\text { Substanz } & R_{f} \text {-Wert in } & \text { S1 } & \text { S2 } \\ \text { Fr. } 125 \mathrm{ff} . & & 0,26 & 0,27 \\ \text { Hypoxanthin } & & 0,15 & 0,20\end{array}$

Fr. $150-168$ 3-Methyladenin $[\lambda \max =273 \mathrm{~nm}(155)]$. Fr. $180-240$ Adenin und oder 1-Methyladenin

$[\lambda \max =261 \mathrm{~nm}(200)]$.

$20 \mathrm{mg}(0,15 \mathrm{mMol})$ Adenin und $220 \mathrm{mg}(1,5 \mathrm{mMol})$ NNMG wurden in $8 \mathrm{ml}$ Pufferlösung von $\mathrm{pH} 5,5$ bei Zimmertemperatur stehen gelassen. Nach 6 Tagen Auftrennung von $4 \mathrm{ml}$ an Dowex (wie oben), je $150 \mathrm{Tr}$. $\mathrm{H}_{2} \mathrm{O}$. Nach wiederholter Trennung an Dowex 50 (wie oben), je 150 Tr. $\mathrm{H}_{2} \mathrm{O}$ Fr. $101-120,2-n$. HCl Fr. $121-130,3-n$. HCl Fr. $131-180$.

Fr. $137-143$ 3-Methyladenin $[\lambda \max =273 \mathrm{~nm}$ (140)].

Fr. $145-170$ 1-Methyladenin + Adenin $[\lambda \max =$ $261 \mathrm{~nm}(150)]$.

Adenosin: $20,0 \mathrm{mg} \quad(0,07 \mathrm{mMol})$ Adenosin wurden in $5,5 \mathrm{ml}$ Puffergemisch von $\mathrm{pH} 5,5$ gelöst, mit $110 \mathrm{mg}$ $(0,75 \mathrm{mMol})$ NNMG versetzt und bei Zimmertemperatur stehengelassen. Die fast farblose Reaktionslösung wurde mit Methanol versetzt, bis sie davon ungefähr $60 \%$ enthielt, und der Trennung unterworfen an Dowex 1X8 $(100-200 \mathrm{mesh}), \mathrm{OH}^{\ominus} / 60$-proz. Methanol, 1,5 20 $\mathrm{cm})$.

Fr. $26-461$-Methyladenosin: $\lambda \max =259 \mathrm{~nm}\left(\mathrm{H}_{2} \mathrm{O}\right)$, $\lambda \max =257 \mathrm{~nm}(\mathrm{n} / 10 \mathrm{HCl})$.

Dünnschichtchromatographie

\begin{tabular}{|c|c|c|}
\hline Substanz & $R_{f}$-Wert an $\mathrm{MN} 400 / \mathrm{S} 4$ & $\mathrm{DEAE} / \mathrm{S} 5$ \\
\hline $\begin{array}{l}\text { Fr. } 26-46 \\
\text { Adenosin }\end{array}$ & $\begin{array}{l}0,60 \\
0,33\end{array}$ & $\begin{array}{l}0,70 \\
0,38\end{array}$ \\
\hline
\end{tabular}

Massenspektrum von Fr. 26-47: $m / e=168(3,5 \%)$, $167(34 \%), 150(8 \%), 149(100 \%), 113(13 \%)$, $112(11 \%), 104(8 \%), 84(5 \%), 83(11 \%), 71$ (27\%), 70 (33\%), 69 (9\%).

$100,5 \mathrm{mg}(0,36 \mathrm{mMol})$ Adenosin wurden mit 549,7 mg $(3,7 \mathrm{mMol})$ NNMG in $11 \mathrm{ml}$ Pufferlösung von $\mathrm{pH}$ 5,5 bei Zimmertemperatur und Lichtabschluß gehalten. Nach spätestens 14 Tagen war alles NNMG verbraucht. Trennung an Dowex 1X8 $\left(100-200 \mathrm{mesh}, \mathrm{OH}^{\ominus} / \mathrm{H}_{2} \mathrm{O}\right.$,

$1,5 \cdot 14 \mathrm{~cm})$, Fraktionen ca. $10 \mathrm{ml}$; wäßrige Lösung wurde aufgezogen und mit steilem Gradienten $(70 \mathrm{ml})$ auf 60-proz. Methanol gebracht. Ab Fr. 71 wurde mit $50 \mathrm{ml} \mathrm{H} \mathrm{H}_{2} \mathrm{O}$ äquilibriert und die Elution mit 0,2-m. $\mathrm{NH}_{4} \mathrm{HCO}_{3}$ fortgesetzt. Fr. $99-106$ Inosin.

Dünnschichtchromatographie an MN 400

Substanz

Fr. 99-106

Inosin

$80 \mathrm{mg}(0,03 \mathrm{mMol})$ Adenosin wurden in $10 \mathrm{ml}$ Puf ferlösung $\mathrm{pH} 5,5$ mit $40 \mathrm{mg}(0,27 \mathrm{mMol})$ NNMG bei $37^{\circ}$ gehalten. Nach 5 Tagen Trennung an Dowex 1X8 $\left(100-200\right.$ mesh, $\left.\mathrm{Cl}^{\ominus} / \mathrm{H}_{2} \mathrm{O}, 1,5 \cdot 11,3 \mathrm{~cm}\right)$ mit $\mathrm{NH}_{3}$. Lösung von $\mathrm{pH} 10$. Fraktionen zu $10 \mathrm{ml}$. Fraktionen 9 bis 46, Rechromatographie an Dowex $50 \mathrm{WX12}(200$ bis 400 mesh, $\mathrm{H}^{\oplus} / 2-n$. $\left.\mathrm{HCl}, 2 \cdot 7 \mathrm{~cm}\right)$, Fraktionen zu $10 \mathrm{ml}$ :

Fr. $6-8 \quad$ Nitroguanidin $(\lambda \max 264 \mathrm{~nm})$,

Fr. 9-11 Hypoxanthin $(\lambda \max 248 \mathrm{~nm})$,

Fr. $60-1401-$ Methyladenin $(\lambda \max 260 \mathrm{~nm})$.

Adenosinmonophosphat: $10 \mathrm{mg}(0,03 \mathrm{mMol})$ Adenosinmonophosphat wurden bei $37^{\circ}$ in $4 \mathrm{ml}$ Puffergemisch vom $\mathrm{pH} 5,5 \mathrm{mit} 50 \mathrm{mg}(0,34 \mathrm{mMol})$ NNMG umgesetzt. Nach Vortrennung an Dowex 1X8 (100-200 mesh, $\left.\mathrm{OH}^{\ominus} / \mathrm{H}_{2} \mathrm{O}, 1,5 \cdot 20 \mathrm{~cm}\right)$ Trennung an Dowex $50 \mathrm{WX} 12$ $\left(200-400\right.$ mesh, $\left.\mathrm{H}^{\oplus} / \mathrm{H}_{2} \mathrm{O}, 2 \cdot 7 \mathrm{~cm}\right)$, Fraktionen zu $10 \mathrm{ml}$ : Fr. $16-19$ Desaminierungsprodukt $(\lambda \max =$ $246-252 \mathrm{~nm})$.

Guanin: $20,6 \mathrm{mg}(0,14 \mathrm{mMol})$ Guanin wurden mit $202 \mathrm{mg}(1,37 \mathrm{mMol})$ NNMG in $4 \mathrm{ml}$ Pufferlösung vom $\mathrm{pH} 5,5$ drei Stdn. lang auf $80^{\circ} \mathrm{C}$ erhitzt.

Dünnschichtchromatographie

Substanz $\quad R_{f}$-Wert an MN 400/S1 S4 DEAE/S5

Lösung $\quad 0,20 \quad 0,30 \quad 0,02$

$\begin{array}{llll}\text { Xanthin } \quad 0,20 & 0,30 & 0,02\end{array}$

UV-Spektrum von $R_{f} 0,30(\mathrm{MN} \mathrm{400/S4)}: \lambda \max =$ $267 \mathrm{~nm}\left(\mathrm{H}_{2} \mathrm{O}\right), \lambda \max =261 \mathrm{~nm}(\mathrm{n} / 10 \mathrm{HCl})$.

Guanosin: $50 \mathrm{mg}(0,18 \mathrm{mMol})$ wurden in $10 \mathrm{ml}$ Pufferlösung von $\mathrm{pH} 5,5 \mathrm{mit} 260 \mathrm{mg}(1,77 \mathrm{mMol}) \mathrm{NNMG}$ 4 Tage auf $37^{\circ}$ gehalten.

Dünnschichtchromatographie an MN 400

$\begin{array}{lrc}\text { Substanz } & R_{f} \text {-Wert in S4 } & \text { S7 } \\ \text { Nitroguanidin } & 0,65 & 0,76 \\ \text { 1-Methyl-3-nitroguanidin } & 0,80 & 0,85 \\ \text { Guanosin } & 0,30 & 0,60 \\ \begin{array}{l}\text { Substanz mit heller Fuoreszenz } \\ \quad \text { im UV-Licht (254 nm) }\end{array} & 0,13 & 0,40\end{array}$

Säulenchromatographie der hydrolysierten Probe an Dowex $50 \mathrm{WX12}\left(200-400 \mathrm{mesh}, \mathrm{H}^{\oplus} 12-n\right.$. HCl, $2 \cdot 7$ cm) Fraktionen zu $10 \mathrm{ml}$ : Fr. $4-10$ enthalten Nitroguanidin und 1-Nitro-3-methyl-guanidin, 23-40 Guanin, 46-62 7-Methyl-guanin. 


\begin{tabular}{|c|c|c|c|c|}
\hline \multirow[t]{2}{*}{ Substanz } & \multicolumn{4}{|c|}{$R_{f}$-Werte und Aktivität in IpM } \\
\hline & S1 & S3 & S8 & S9 \\
\hline Adenin & 0,50 & 0,26 & 0,50 & 0,48 \\
\hline 1-Methyladenin & $\begin{array}{l}0,15 \\
3112\end{array}$ & $\begin{array}{r}0,68 \\
308\end{array}$ & $\begin{array}{l}0,27 \\
51,9 \pm 1,1\end{array}$ & $\begin{array}{l}0,32 \\
236\end{array}$ \\
\hline 3-Methyladenin & $\begin{array}{r}0,36 \\
356\end{array}$ & $\begin{array}{l}0,61 \\
36,6 \pm 1,1\end{array}$ & $\begin{array}{r}0,40 \\
198\end{array}$ & $\begin{array}{r}0,15 \\
347\end{array}$ \\
\hline $\mathrm{N}^{6}$-Methyladenin & $\begin{array}{r}0,68 \\
164\end{array}$ & $\begin{array}{l}0,35 \\
21,9 \pm 1,1 \\
6,0 \pm 0,9 *\end{array}$ & $\begin{array}{l}0,73 \\
13,9 \\
\text { 土1,0 }\end{array}$ & $\begin{array}{r}0,70 \\
101\end{array}$ \\
\hline $\begin{array}{l}\text { 1-Nitro-3-methyl- } \\
\text { guanidin }\end{array}$ & $\begin{array}{c}0,63 \\
52470\end{array}$ & $\begin{array}{l}0,81 \\
2136\end{array}$ & $\begin{array}{l}0,66 \\
2650\end{array}$ & $\begin{array}{r}0,64 \\
36263\end{array}$ \\
\hline
\end{tabular}

Tab. 1. Methylierungsprodukte nach Behandlung von Adenin mit ${ }^{14} \mathrm{C}$-Methyl-NNMG bei pH 6 und $37^{\circ}$ in Citronensäure-Phosphat-Puffer.

\begin{tabular}{|c|c|c|c|c|}
\hline Substanz & $\mathrm{S}_{\mathrm{S}} R^{R_{f}-\mathrm{W}}$ & $\begin{array}{l}\text { d Aktivität } \\
\text { S3 }\end{array}$ & S8 & S9 \\
\hline Adenin & 0,45 & 0,26 & 0,45 & 0,48 \\
\hline 1-Methyladenin & $\begin{array}{l}0,14 \\
72\end{array}$ & $\begin{array}{l}0,62 \\
10,9 \pm 0,9\end{array}$ & $\begin{array}{l}0,21 \\
15,5 \pm 1,0\end{array}$ & $\begin{array}{c}0,34 \\
10,1 \pm 0,9\end{array}$ \\
\hline 3-Methyladenin & $\begin{array}{l}0,38 \\
4,0 \pm 0,8 *\end{array}$ & $\begin{array}{l}0,62 \\
10,9 \pm 0,9\end{array}$ & $\begin{array}{l}0,35 \\
11,5 \pm 1,3\end{array}$ & $\begin{array}{c}0,15 \\
10,6 \pm 0,9\end{array}$ \\
\hline $\mathrm{N}^{6}$-Methyladenin & $\begin{array}{l}0,68 \\
7,9 \pm 1,2\end{array}$ & $\begin{array}{l}\mathbf{0 , 3 4} \\
\mathbf{3 , 6} \pm 0,7\end{array}$ & $\begin{array}{l}0,63 \\
3,9 \pm 0,6\end{array}$ & $\begin{array}{l}0,70 \\
8,8 \pm 0,9\end{array}$ \\
\hline
\end{tabular}

Tab. 2. Methylierungsprodukte nach Behandlung von Poly-A (Bedingungen wie Tab. 1). * Durch Rechromatographie erhaltene Werte.

$10 \mathrm{mg}(0,035 \mathrm{mMol})$ Guanosin und $52 \mathrm{mg} \quad(0,35$ mMol) NNMG wurden in $10 \mathrm{ml}$ Pufferlösung von $\mathrm{pH}$ 5,5 5 Tage bei $37^{\circ}$ gehalten. Die Trennung des Reaktionsgemischs erfolgte an Dowex 50 (wie oben), Fraktionen zu $10 \mathrm{ml}, \mathrm{H}_{2} \mathrm{O} 1-20,2-n$. $\mathrm{HCl} 21 \mathrm{ff} .:$ Fr. $2-13$ Nitroguanidin und 1-Nitro-3-methylguanidin, 23-33 Guanosin und Xanthin, $35-55$ Guanin.

$20 \mathrm{mg}(0,07 \mathrm{mMol})$ Guanosin in 5,2 ml Pufferlösung von $\mathrm{pH}$ 5,5 wurden bei Zimmertemperatur der wäßrigen Lösung von $104 \mathrm{mg}(0,70 \mathrm{mMol})$ NNMG ausgesetzt.

Dünnschichtchromatographie

\begin{tabular}{lrlc} 
Substanz $\quad R_{f}$-Wert an MN $400 / \mathrm{S} 1$ & \multicolumn{1}{l}{ S4 } & DEAE/S5 \\
Reaktionslösung & 0,03 & 0,17 & 0,01 \\
Xanthosin & 0,03 & 0,17 & 0,01
\end{tabular}

Guanosinmonophosphat: $8 \mathrm{mg}(0,022 \mathrm{mMol})$ Guanosinmonophosphat wurden in $10 \mathrm{ml}$ Puffergemisch vom $\mathrm{pH} 5,5 \mathrm{mit} 40 \mathrm{mg}(0,27 \mathrm{mMol})$ NNMG umgesetzt und 5 Tage a) bei Zimmertemperatur, b) bei $37{ }^{\circ} \mathrm{C}$ gehalten. Auftrag der Reaktionsprodukte an einer Säule von Dowex $50 \mathrm{WX12}\left(200-400 \mathrm{mesh}, \mathrm{H}^{\oplus} / 2-n\right.$. HCl, $2 \cdot 7$ $\mathrm{cm})$ und Prüfung bei $260 \mathrm{~nm}$ : a) Fr. $48-58$ flacher und b) Fr. 44-59 deutlicher Absorptionspeak von 7-Methylguanin.
Einwirkung von ${ }^{14} \mathrm{C}-\mathrm{Methyl-NNMG}$ a uf Nucleobasen bzw. Nucleinsäuren

Darstellung von ${ }^{14} \mathrm{C}$-Methyl-NNMG (in Anlehnung an die Vorschrift von $\mathrm{McKay}{ }^{12}$ ) : $2 \mathrm{mc}^{14} \mathrm{C}$-Methylaminhydrochlorid (spez. Aktivität ca. $30 \mathrm{mc} / \mathrm{mMol}$ ) gelöst in Äthanol, wurden unter Eiskühlung mit einigen Tropfen konz. NNMG-Lösung versetzt. $40 \mathrm{mg}(1,3 \mathrm{mMol})$ Methylamin wurden als 40-proz. methanolische Lösung zugetropft und dazu soviel festes NNMG zugegeben, $\mathrm{da}$ ß sich insgesamt $250 \mathrm{mg}(1,7 \mathrm{mMol})$ davon im Reaktionsgefäß befanden. Nach Beendigung der Gasentwicklung sind die Kristalle von 1-Nitro-3-methyl-guanidin abfiltriert worden. Die Substanz wurde in einem kleinen $\mathrm{Erlen}$ meyer-Kolben in $0,18 \mathrm{ml} \mathrm{HNO}_{3}(d=$ $1,42)$ aufgelöst. Die Lösung ist dann mit $0,8 \mathrm{ml} \mathrm{H}_{2} \mathrm{O}$ verdünnt worden. Unter ständigem Rühren wurden $80 \mathrm{mg}(1,16 \mathrm{mMol}) \mathrm{NaNO}_{2}$, gelöst in $0,12 \mathrm{ml} \mathrm{H} \mathrm{H}_{2} \mathrm{O}$, in 5 Min. zugegeben. Nach 20 Min. langem Rühren sind die gelben Kristalle des ${ }^{14} \mathrm{C}$-Methyl-NNMG abfiltriert worden (spez. Aktivität $2 \mathrm{mc} / \mathrm{mMol}$ ).

Umsetzung der Nucleobasen bzw. der Nucleinsäuren mit ${ }^{14}$ C-Methyl-NNMG: Als Reaktionslösung diente Phosphat-Citrat-Puffer von $\mathrm{pH}$ 6. $1 \mathrm{mg}$ Base (Adenin oder Cytosin) bzw. $1 \mathrm{mg}$ Nucleinsäure (Poly-A, Poly-C, Poly-UG) und $0,3-1,0 \mathrm{mg}{ }^{14} \mathrm{C}$-Methyl-NNMG in $1 \mathrm{ml}$

12 A. F. McKay, J. Amer. chem. Soc. 71, 1967 [1949]. 
Pufferlösung wurden $24-48 \mathrm{Stdn}$. bei $37{ }^{\circ} \mathrm{C}$ geschüttelt. Die Nucleinsäuren wurden ungefähr $10 \mathrm{Stdn}$. lang durch Dialyse gegen deion. Wasser gereinigt. Darauf wurden Poly-A und Polv-UG mit 1-n. $\mathrm{HCl}$ bei $100^{\circ}$ 2 Stdn., Poly-C mit 20-proz. HCl im zugeschmolzenen Rohr bei $120^{\circ} 2 \mathrm{Stdn}$. hydrolysiert.

Identifizierung der Methylierungsprodukte: Die Identifizierung auf den Dünnschichtplatten wurde durch Co-Chromatographie mit Vergleichssubstanzen verschiedener Herkunft an MN 400 in mehreren Laufmitteln vorgenommen. Die bei $254 \mathrm{~nm}$ löschenden Substanzflecken wurden ausgekratzt und nach Rechromatographie in einem anderen Laufmittel die Radioaktivität mit $7 \mathrm{ml}$ Dioxan-Szintillator in einem Packard-Scintillation-Spectrometer gemessen.

\begin{tabular}{|c|c|c|c|}
\hline Substanz & $\begin{array}{l}R_{\mathrm{f}}-\text { Werte } \\
\quad \mathrm{S} 2\end{array}$ & $\begin{array}{l}\text { d Aktivität } \\
\text { S3 }\end{array}$ & $\mathrm{IpM}_{\mathrm{S} 10}$ \\
\hline Guanin & 0,14 & 0,46 & 0,09 \\
\hline $\begin{array}{l}\text { 7-Methyl- } \\
\text { guanin }\end{array}$ & $\begin{array}{c}0,19 \\
9,1 \pm 0,8\end{array}$ & $\begin{array}{c}0,64 \\
9,6 \pm 0,9\end{array}$ & $\begin{array}{c}0,17 \\
7,3 \pm 0,8\end{array}$ \\
\hline
\end{tabular}

Tab. 3. Methylierungsprodukt nach Behandlung von Poly-UG (Bedingungen wie Tab. 1).

\begin{tabular}{lcccc}
\hline \multirow{1}{*}{ Substanz } & \multicolumn{4}{c}{$R_{\mathrm{f}}$-Werte und Aktivität in IpM } \\
& $\mathrm{S} 1$ & $\mathrm{~S} 2$ & $\mathrm{~S} *^{*}$ & $\mathrm{~S} 10$ \\
\hline Cytosin & 0,20 & 0,39 & 0,70 & 0,24 \\
$\begin{array}{l}\text { 3-Methyl- } \\
\text { cytosin }\end{array}$ & 0,26 & 0,55 & 0,77 & 0,42 \\
& 382 & $36,1 \pm 1,2$ & & $29,5 \pm 1,1$ \\
$\begin{array}{l}\text { 1-Methyl- } \\
\text { cytosin }\end{array}$ & $47,4 \pm 1,2$ & & & \\
& 0,15 & 0,64 & 0,84 & 0,50 \\
$\begin{array}{l}\text { 1-Nitro-3- } \\
\text { methyl- }\end{array}$ & $7,3 \pm 0,6$ & $8,3 \pm 0,9$ & & $43,2 \pm 1,2$ \\
guanidin & 0,60 & 0,78 & 0,70 & 0,58 \\
\hline
\end{tabular}

* System S 3 diente zur Vortrennung, Rechromatographie in den übrigen Systemen.

Tab. 4. Methylierungsprodukte nach Behandlung von Cytosin (Bedingungen wie Tab. 1).

\begin{tabular}{lcccc}
\hline Substanz & \multicolumn{4}{c}{$R_{\mathrm{P}}$-Werte und Aktivität in IpM } \\
& $\mathrm{S} 1$ & $\mathrm{~S} 2$ & $\mathrm{~S} 3$ & $\mathrm{~S} 10$ \\
\hline Cytosin & 0,20 & 0,38 & 0,70 & 0,24 \\
l-Methyl- & 0,15 & 0,64 & 0,84 & 0,49 \\
cytosin & $19,8 \pm 1,0$ & $\mathbf{2 7 , 8} \pm 0,4$ & $\mathbf{9 2 , 7} \pm 0,8$ & $\mathbf{5 3 , 4} \pm 0,8$
\end{tabular}

Tab. 5. Methylierungsprodukt nach Behandlung von Poly-C (Bedingungen wie Tab. 1).

13 C. H. Decker, J. Amer. chem. Soc. 87, 4027 [1965].

\section{Ergebnisse und Diskussion}

\section{Derivate des Adenins}

Wird Adenin bei pH 5,5 bei Zimmertemperatur und bei $80^{\circ}$ mit NNMG bis zur Entfärbung des Mutagens umgesetzt, so lassen sich nach säulenchromatographischer Trennung 1-Methyladenin und 3-Methyladenin isolieren. Der Nachweis erfolgte durch die UV-Maxima bei $261 \mathrm{~nm}$ bzw. $273 \mathrm{~nm}$ und durch Dünnschichtchromatographie in 2 Systemen. Nach einer Umsetzung des Adenosins bei Zimmertemperatur und $37^{\circ}$ mit NNMG wurde nach Auftrennung nach dem Verfahren von Decker ${ }^{13}$ 1-Methyladenosin durch das UV-Maximum bei $259 \mathrm{~nm}$ und massenspektrometrisch durch die Massenzahl 149 nachgewiesen. Inosin konnte dünnschichtchromatographisch identifiziert werden. Nach Hydrolyse wurden Hypoxanthin und 1-Methyladenin sowohl dünnschichtchromatographisch in zwei Laufmitteln als auch durch die UV-Maxima bei $248 \mathrm{~nm}$ bzw. $260 \mathrm{~nm}$ nachgewiesen. Aus Adenosinmonophosphat war Hypoxanthin mit ca. 1\% Ausbeute zu erhalten. Bei der Umsetzung von Adenin mit ${ }^{14} \mathrm{C}-$ Methyl-NNMG konnten neben 1-Methyladenin und 3-Methyladenin auch $N^{6}$-Methyladenin durch Co-Chromatographie mit Vergleichssubstanzen und Messung der Radioaktivität nachgewiesen werden (Tab. 1). Diese Verbindungen traten unter gleichen Reaktionsbedingungen auch bei einer entsprechenden Umsetzung mit Polyadenylsäure auf (Tab. 2). 3-Methyladenin wurde kürzlich auch von LAwLEY ${ }^{14}$ in NNMG-behandelter DNS nachgewiesen.

\section{Derivate des Guanins}

Nach der Umsetzung des Guanins mit NNMG sowohl bei $80^{\circ}$ als auch bei Zimmertemperatur wurde Xanthin dünnschichtchromatographisch und durch das UV-Spektrum identifiziert. Aus Guanosin konnte nach entsprechender Reaktion bei Zimmertemperatur bzw. $37^{\circ}$ und bei pH 5,5 Xanthosin mit Hilfe der Dünnschichtchromatographie nachgewiesen werden. 7-Methylguanosin gab sich durch helle Fluoreszenz im UV-Licht bei $254 \mathrm{~nm}$ auf der Dünnschichtplatte zu erkennen, und die Fluoreszenz ließ sich im Gegensatz zu der des Guanosins durch Ammoniakdämpfe nicht löschen. Nach Hydrolyse und Trennung an Dowex 50 wurden außerdem Xanthin und 7-Methylguanin gefunden. 7-Methylguanin ließ sich

14 P. D. Lawley, Nature [London] 218, 580 [1968]. 
$\overline{\text { Ausgangssubstanz (Reaktionspartner Reaktionsprodukte }}$ $\operatorname{des} \mathrm{NNMG}$ )

Derivate des Adenins

Adenin, Adenosin, Adenosinmonophosphat, Polyadenylsäure

Adenin, Adenosin, Addenosinmonomonophosphat, Polyadenylsäure

Adenin, Polyadenylsäure

Adenin, Adenosin, Adenosinmono-

phosphat

Derivate des Guanins

Guanosin, Guanosinmonophosphat, Polyuridylguanylsäure

Guanin, Guanosin

Derivate des Cytosins

Cytosin, Polycytidylsäure

Cytosin
1-Methyladenin

3-Methyladenin

$N^{6}$-Methyladenin

Hypoxanthin

7-Methylguanin

Xanthin

1-Methylcytosin 3-Methylcytosin
Tab. 6. Umsetzungsprodukte der Nucleobasen und ihrer Derivate mit NNMG.

auch nach einer Reaktion von Guanosinmonophosphat mit NNMG nach Hydrolyse und Trennung über Dowex 50 erhalten. Mit Hilfe von ${ }^{14}$ C-Methyl-NNMG wurde 7-Methylguanin aus Polyuridylguanylsäure nach Dialyse, Hydrolyse und Chromatographie gefunden (Tab. 3) . 7-Methylguanin ist in jüngster Zeit von LAwLEY in der DNS nach Behandlung mit NNMG festgestellt worden ${ }^{14}$. Ein Nachweis dieser Verbindung in vivo gelang CRADDock ${ }^{15}$.

\section{Derivate des Cytosins}

Im Gegensatz zu den Ergebnissen mit Purinbasen ließ sich nach einer Reaktion bei pH 5,5 mit NNMG sowohl bei Cytosin als auch bei Cytidin weder bei Zimmertemperatur noch bei $80^{\circ}$ das erwartete Desaminierungsprodukt Uracil nachweisen. Dies stimmt mit Ergebnissen unserer Untersuchungen des Einflusses von NNMG auf die Matrizenaktivität der Homopolyribonucleotide überein ${ }^{1}$. 1-Methylcytosin und 3-Methylcytosin ließen sich in einem Reaktionsgemisch von Cytosin mit ${ }^{14} \mathrm{C}$-Methyl-NNMG bei pH 6 und $37^{\circ}$ durch Co-Chromatographie nachweisen (Tab. 4). Im Hydrolysat der umgesetzten Polycytidylsäure konnte auf die gleiche Weise 1-Methylcytosin identifiziert werden (Tab. 5).

\section{Zum Reaktionsmechanismus}

Für die Methylierungs- und Desaminierungsreaktion des NNMG sind hauptsächlich zwei Reaktionen

15 V. M. Craddock, Biochem. J. 106, 921 [1968].

16 P. Emmelot, I. J. Mizrahi u. E. Kriek, Nature [London] 193, 1158 [1962]. zu erwägen. Das NNMG-Molekül bietet Hydroxylionen zwei Angriffspunkte, am N-Atom der Nitrosogruppe (1) und am Guanidin-C-Atom (2). Die eine Reaktion (1), für die auch ein elektrophiler Angriff eines $\mathrm{H}^{\oplus}$ am $\mathrm{N}^{1}$ in Frage kommt, führt zu 1-Nitro3-methyl-guanidin und salpetriger Säure bzw. Nitrit, wie sich beim Stehenlassen in Lösung zeigt. Die zweite Reaktion (2) bedingt die Bildung des methylierenden Agens, das vermutlich über ein Methylnitrosamin, Methyldiazohydroxyd bzw. Methyldiazotat entsteht. Die Bildung des nicht faßbaren Nitroharnstoffs ist aus dem Nitroguanylrest herzuleiten, der seinerseits Nitroguanylierungen an den Nucleobasen vornehmen könnte. Entstandener Nitroharnstoff kann sich entweder selbst mit NNMG sofort umsetzen, wie in Versuchen festgestellt werden konnte, oder in Form seiner Hydrolyseprodukte. Hierbei entsteht vermutlich das von uns nachgewiesene Nitroguanidin.

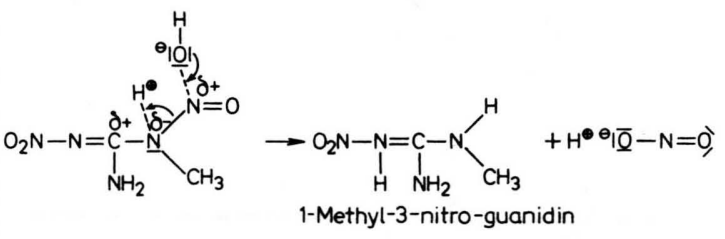

(1)



Die Bildung eines Methyldiazohydroxyds nehmen Еммецот et al. ${ }^{16}$ als Zwischenprodukt bei der Methylierung durch $N$-Nitroso- $N$-methylurethan an $(2,2)$. Monomethylnitrosamin könnte dabei intermediär auftreten. Ein Reaktionsmechanismus für NNMG über Diazomethan ist nach unseren Versuchen bei pH 5,5-6,0 wenig wahrscheinlich, da sich Diazomethan lediglich bei $\mathrm{pH} 13$ und 14 nachweisen läßt. Es ist allerdings von MeErwein ${ }^{17}$ eine alkoholytische Spaltung von $N$-Nitroso- $N$-methylurethan bekannt, bei der unter neutralen Bedingungen „naszie-

17 Patent der Schering-Kahlbaum AG, Erfinder H. Meerwein, C. 1933 II 1758 [1933]. 
rendes Diazomethan“ mit Aldehyden und Ketonen reagieren soll. Henry ${ }^{18}$ wies aber daraufhin, daß bei NNMG unter den von McKAY gewählten Versuchsbedingungen, einer Umsetzung in wäßriger Kalilauge ${ }^{19}$, die Bildung von $N$-Methyl-anilin über einen Diazomethanmechanismus nicht erklärt werden könne. Nach Versuchen von Kriek und EmmeLOT ${ }^{20}$ werden Nucleinsäuren auch durch ein Gemisch von Methylamin und salpetriger Säure bei $\mathrm{pH} 4,0$ methyliert. In diesem Reaktionsgemisch wird nach Austiv ${ }^{21}$ über das Methyldiazoniumion ein Methylkation freigesetzt, das die Nucleobasen methyliert. Vermutlich trifft daher vor allem im sauren und neutralen Bereich für NNMG Reaktion 2,2a zu. Hier entsteht das Methyldiazoniumion, das unter Stickstoffabspaltung das elektrophile Methylkation freisetzen könnte. Durch jüngste Untersuchungen von Lisinsky, Loo und Ross ${ }^{22}$ mit $\mathrm{C}^{2} \mathrm{H}_{3}$-Dimethylnitrosamin an Ratten scheint diese Hypothese gestützt zu werden. Sie zeigten, daß eine Methylierung zu 7-Methylguanin über Diazomethan für dieses Nitrosamin nicht in Frage kommt.

18 R. A. Henry, J. Amer. chem. Soc. 72, 3287 [1950).

19 A. F. McKay, J. Amer. chem. Soc. 70, 1974 [1948].

20 E. Kriek u. P. Emmelot, Biochim. biophysica Acta [Amsterdam] 91, 59 [1964].

21 A. T. Austin, Nature [London] 188, 1086 [1960].

22 W. Lijinsky, J. Loo u. A. E. Ross, Nature [London] 218, 1174 [1968].
Nach unseren Ergebnissen scheint für die Mutationsauslösung durch NNMG eine Methylierung in der Zelle nicht allein auf Grund einer einfachen Reaktion von NNMG mit den Nucleobasen vorzuliegen. Offensichtlich sind am Mechanismus der Methylierung Sulfhydryl-Verbindungen beteiligt, da in Gegenwart von SH-Verbindungen eine erhebliche Beschleunigung der Methylierungsreaktion beobachtet werden konnte ${ }^{23,24}$.

Die Nitroguanylierung spielt wahrscheinlich bei der Mutationsauslösung auch eine gewisse Rolle.

In diesem Zusammenhang ist erwähnenswert, daß wir bei der Umsetzung von Phenol mit NNMG $O$. Phenylisonitroharnstoff und entsprechend aus $p$-Kresol $O$ - $p$-Kresyl-isonitroharnstoff erhalten haben ${ }^{25}$.

Dem Fonds der Chemischen Industrie und der Deutschen Forschungsgemeinschaft danken wir für Unterstützung der Arbeit, Herrn Dr. J. Heiss und Herrn K. P. Zeller (Tübingen) danken wir für Aufnahme und Interpretation von Massenspektren.

${ }^{23}$ R. Süssmuth u. F. Lingens, Naturwissenschaften 55, 85 [1968].

24 R. Süssmuth u. F. Lingens, Veröffentlichung in Vorbereitung.

${ }^{25}$ F. Lingens u. J. RaU, unveröffentlicht. 